\title{
Status Of Super Heavy Element Synthesis Experiments
}

Mark Stoyer*i

Lawrence Livermore National Laboratory, Livermore, California 94551, USA

E-mail: mastoyer@llnl.gov

\section{N.J. Stoyer}

Lawrence Livermore National Laboratory, Livermore, California 94551, USA

\section{J.H. Hamilton}

Department of Physics and Astronomy, Vanderbilt University, Nashville, Tennessee 37235, USA

E-mail: j.h.hamilton@Vanderbilt.Edu

\section{S.Y. Strauss}

Department of Physics, University of Notre Dame, Notre Dame, Indiana 46566, USA

E-mail: sstrauss@nd.edu

During the last 18 years, six new elements have been discovered and confirmed and over 50 new isotopes have been synthesized in nuclear reactions using ${ }^{48} \mathrm{Ca}$ beams and actinide targets $\left({ }^{237} \mathrm{~Np},{ }^{239,240,242,244} \mathrm{Pu},{ }^{243} \mathrm{Am},{ }^{245,248} \mathrm{Cm},{ }^{249} \mathrm{Bk},{ }^{249,251} \mathrm{Cf}\right.$.) This work has resulted in a wealth of nuclear decay data and some nuclear structure information on the heaviest isotopes and decay progeny which will be discussed. The extent of the region of enhanced stability near $Z=114$ and $N=184$ is not completely known. To attempt to produce the heaviest isotopes of element 118 , new experiments were performed using ${ }^{48} \mathrm{Ca}$ projectiles and a ${ }^{251} \mathrm{Cf}$ (mixed isotope) target at the Dubna Gas Filled Recoil Separator (DGFRS) located in the Flerov Laboratory of Nuclear Reactions in Dubna. The probability that any observed decay chains are due to random events occurring in the detectors or electronics rather than correlated decay chains, using the LLNL-developed method of Monte Carlo Random Probability analysis will be discussed. This probability will be discussed for recent ${ }^{48} \mathrm{Ca}+{ }^{239,240} \mathrm{Pu}$ experiments in addition to the element 118 experiment and will be compared with other techniques for estimating such probabilities in super heavy element production reactions in which few decay chains are observed.

The 26th International Nuclear Physics Conference

11-16 September, 2016

Adelaide, Australia

\footnotetext{
* Speaker.

${ }^{\dagger}$ This work performed under the auspices of the U.S. Department of Energy by Lawrence Livermore National Laboratory under Contract DE-AC52-07NA27344.
} 


\section{Introduction}

Since 1998, six new chemical elements and over 50 new isotopes have been added to the chart of nuclides in the region of the heaviest nuclei $[1,2,3,4]$. The new isotopes with their measured half-lives and nuclear decay modes are shown in Figure 1. The region is dominated by alphadecay and spontaneous fission (yellow and green boxes, respectively). The production reactions for these various isotopes are indicated in the blue text. The heaviest isotopes observed are $A=294$ nuclei, namely ${ }^{294} \mathrm{Og}$ and ${ }^{294} \mathrm{Ts}$. While it remains experimentally challenging to reach isotopes with the predicted neutron magic number $N=184$ because of no suitable projectile and target combinations, it is important to explore the limits of this region of enhanced stability by attempting to produce both heavier mass and lighter mass isotopes. This paper describes two such attempts utilizing beams of ${ }^{48} \mathrm{Ca}$ : production of lighter isotopes of $\mathrm{Fl}$ using lower mass ${ }^{239,240} \mathrm{Pu}$ targets and production of heavier isotopes of $\mathrm{Og}$ using a target enriched in ${ }^{251} \mathrm{Cf}$. The former reaction has the possible advantage of connecting this region of enhanced stability with "mainland" isotopes should longer decay chains be observed and enabling firmer establishment of $Z$ and $A$ for isotopes in this region, as well as exploring the predicted region of fissioning isotopes. The latter reaction has the possibility of producing the heaviest isotopes ever observed.

Because the cross-sections for producing new isotopes in both reactions are expected to be extremely small, it is important to determine the probability that any observed series of events are due to random events such as fluctuations in background or operating conditions of the experiments, which often run for months at a time. This establishes real correlated decay chains and eliminates those due to random events. This paper includes an analysis of recent experiments and the observed decay chains using the Monte Carlo Random Probability method developed at LLNL [5, 6].

\section{2. ${ }^{48} \mathbf{C a}+{ }^{239,240} \mathbf{P u}$ experiments}

Experiments aimed at the synthesis of lighter isotopes of $\mathrm{Fl}$ were performed using ${ }^{48} \mathrm{Ca}$ beams and ${ }^{239,240} \mathrm{Pu}$ targets and are described in more detail in [7]. The experiments were performed at the Dubna Gas-Filled Recoil Separator (DGFRS) using the ${ }^{48} \mathrm{Ca}$ beam accelerated at the U400 cyclotron of the Flerov Laboratory of Nuclear Reactions, JINR. The ${ }^{48} \mathrm{Ca}$ ion beam was delivered to the target with a maximum intensity of 1.3 particle $\mu \mathrm{A}$ and the beam energy was determined with a systematic uncertainty of $1 \mathrm{MeV}$ by a time-of-flight system placed in front of the DGFRS.

The target materials were provided by Oak Ridge National Laboratory (ORNL) $\left({ }^{240} \mathrm{Pu}\right.$, enrichment of $98.97 \%)$ and JINR $\left({ }^{239} \mathrm{Pu}\right.$ and ${ }^{240} \mathrm{Pu}$, enrichment of $98.2 \%$ and $92 \%$, respectively). The average thicknesses of the targets for the main isotopes were $0.50 \pm 0.05 \mathrm{mg} / \mathrm{cm}^{2}$ for ${ }^{239} \mathrm{Pu}$ and $0.39 \pm 0.04 \mathrm{mg} / \mathrm{cm}^{2}$ for mixed ORNL/JINR ${ }^{240} \mathrm{Pu}$ material in the ratio $1 / 5$ (given uncertainties correspond to standard deviations of thicknesses measured for six sectors of each target). The targets were manufactured by depositing $\mathrm{PuO}_{2}$ oxide onto $0.71-0.72 \mathrm{mg} / \mathrm{cm}^{2}$ Ti foils. Each target had an area of $5.4 \mathrm{~cm}^{2}$ in the shape of an arc segment with an angular extension of $60^{\circ}$ and an average radius of $60 \mathrm{~mm}$. The segments were mounted on a disk that was rotated at $1700 \mathrm{rpm}$ such that the target was perpendicular to the direction of the incoming beam. In the course of the bombardment with the ${ }^{48} \mathrm{Ca}$ beam, the target layers were systematically monitored by counting $\alpha$ particles from the decay of the target isotopes. 


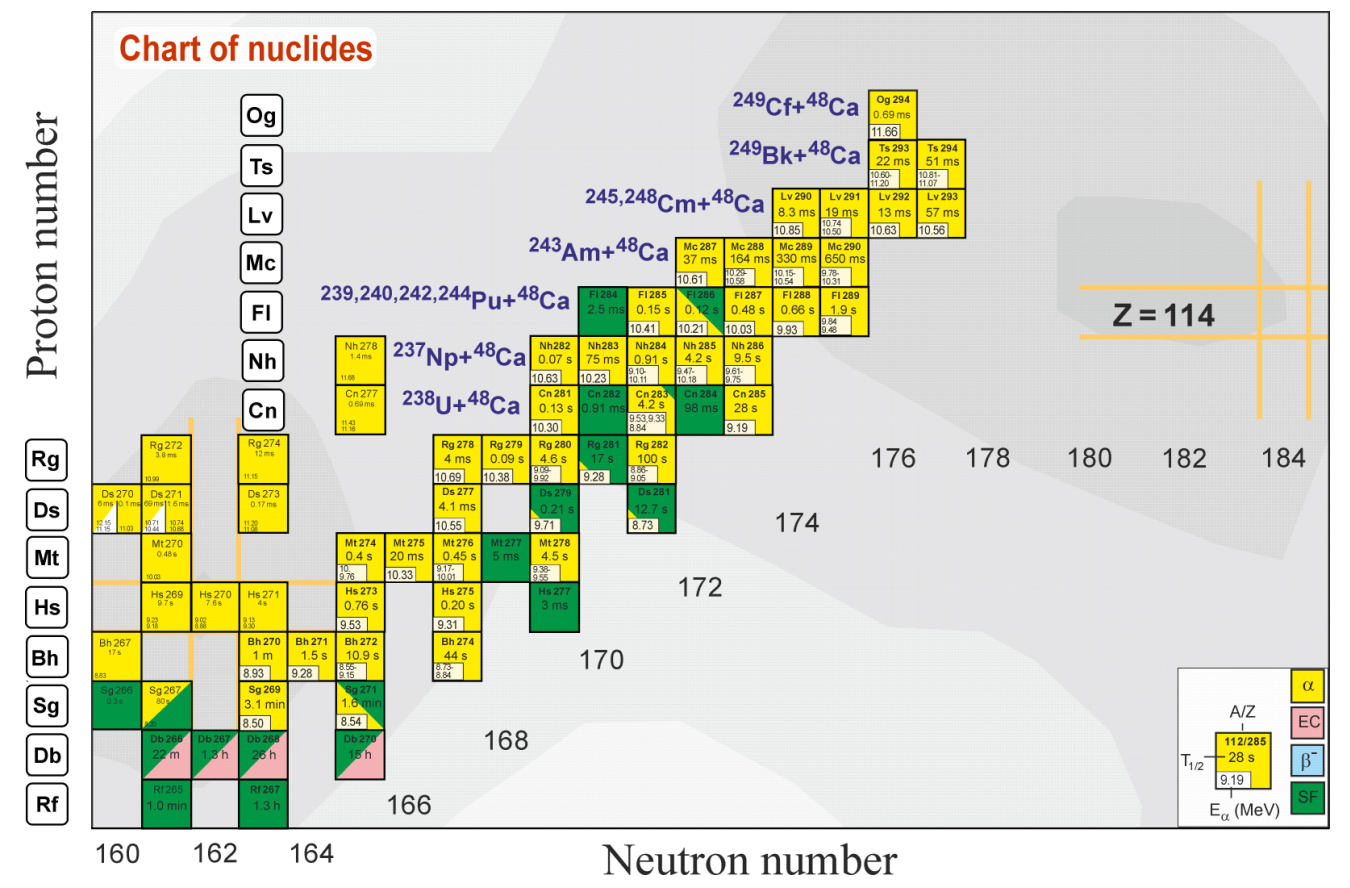

Figure 1: Upper part of the chart of nuclides. Individual boxes represent an isotope and include information on decay mode (colors - see legend), half-life and energies of decay particles. More than 50 isotopes have been produced with ${ }^{48} \mathrm{Ca}$-induced nuclear reactions on various actinide targets with the specific reactions indicated in blue text. The shaded grey regions in the background indicate shell correction energies from a microscopic-macroscopic model and the location of predicted closed shells of $Z=114$ and $N=184$ are indicated with yellow lines.

Experiments were run with laboratory beam energies of $245 \mathrm{MeV}\left({ }^{239,240} \mathrm{Pu}\right.$ targets) and 250 $\mathrm{MeV}\left({ }^{240} \mathrm{Pu}\right.$ target). The total dose for the ${ }^{239} \mathrm{Pu}$ target was $1.4 \times 10^{19}$ and for each of the beam energies used for the ${ }^{240} \mathrm{Pu}$ target about $4 \times 10^{18}$.

Evaporation residues (ERs) recoiling from the target were separated in flight from ${ }^{48} \mathrm{Ca}$ beam ions, scattered particles, and transfer-reaction products by the DGFRS. The transmission efficiency of the separator for $Z=114$ nuclei was estimated to be about $35 \% \pm 5 \%$. Recoils passed through a time-of-flight (TOF) system and were implanted in the detectors. The TOF system consists of two multiwire proportional counters (MWPCs) placed at a distance of $65 \mathrm{~mm}$ between them. The detectors and MWPC are placed in pentane at a pressure of about 1.5 Torr. A $0.2 \mathrm{mg} / \mathrm{cm}^{2}$ Mylar foil separates the detection system from the DGFRS volume, which is filled with hydrogen at a pressure of 1 Torr.

The array of Silicon detectors at the DGFRS final focus was a 0.3-mm-thick double-sided silicon strip detector (DSSD). This large DSSD has 1-mm-wide strips, 48 on the front side and 128 on the back side, creating $61441 \mathrm{~mm}^{2}$ pixels in one silicon wafer. Such high pixilation helps to achieve superior position resolution for recoil-correlated decay sequences reducing potential random events. The recoil implantation counter was surrounded by six single Si detectors, each 500 microns thick and having an active area of $65 \mathrm{~mm}$ by $120 \mathrm{~mm}$. Two pairs of these Si-box 
detectors were mounted at the DSSD long side, and two detectors were used to close the Si-box geometry, one at each short side, forming a detector system akin to a shoebox with the lid removed. All Si counters had a minimum amount of supporting frame material. The detection efficiency for the focal plane DSSD is $52 \%$, but the overall detection efficiency for events escaping the focal plane detector including the Si-box detectors is increased to $85 \%$. A single Si veto detector of 0.5 $\mathrm{mm}$ thickness is mounted behind the DSSD focal plane detector to reject high energy light charged particles produced in direct reactions by the beam with DGFRS materials, which would otherwise mimic $\alpha$-particles.

In the ${ }^{48} \mathrm{Ca}+{ }^{240} \mathrm{Pu}$ experiment at $E_{l a b}=245 \mathrm{MeV}$, we observed three decay chains of ${ }^{285} \mathrm{Fl}$, which is the product of the $3 n$-reaction channel (see Fig. 2). This isotope was first observed in the $5 n$-reaction channel of the ${ }^{48} \mathrm{Ca}+{ }^{242} \mathrm{Pu}$ reaction performed at LBNL [8] and the observed decay sequences are in good agreement with these measurements.

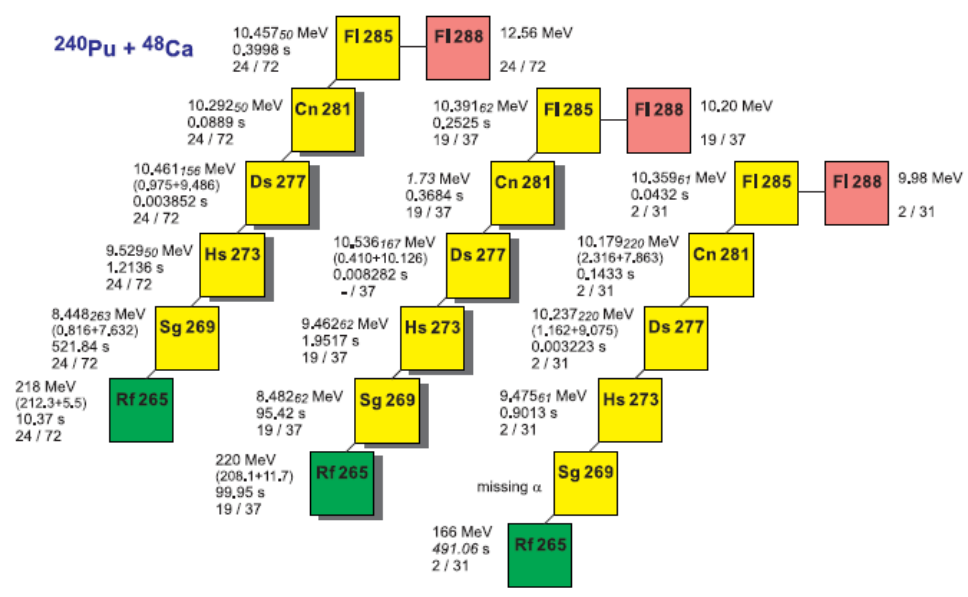

Figure 2: Decay properties of ${ }^{285} \mathrm{Fl}$ and descendant nuclei observed in the ${ }^{48} \mathrm{Ca}+{ }^{240} \mathrm{Pu}$ reaction at $E_{l a b}=$ $245 \mathrm{MeV}$. The upper-right rows for each chain show ER (in pink) energies and strip numbers (front and back). The left row provides energies, time intervals between events, and their strip numbers for alpha decay (in yellow) and SF (in green). Energies of summed signals are given in parenthesis. Events marked with a shadow occured during beam-off periods with lower background. The uncertainties in alpha-particle energies are shown by smaller italic numbers. The time interval for a SF event following a "missing $\alpha^{\text {" was }}$ measured from the proceeding event and is shown in italics. This figure is reproduced from Figure 1 in [7].

The beam energy was increased to $E_{l a b}=250 \mathrm{MeV}$, and four ER-SF events were observed that were not due to fission isomers (see [7] for detailed discussion). These events are shown in Figure 3 together with an additional event that was observed in the ${ }^{48} \mathrm{Ca}+{ }^{239} \mathrm{Pu}$ experiment. These events are tentatively assigned to ${ }^{284} \mathrm{Fl}$, the product of the $4 n$-reacton channel for the experiment with the ${ }^{240} \mathrm{Pu}$ target, and the $3 n$-reaction channel for the lighter mass ${ }^{239} \mathrm{Pu}$ target. This short chain is 
perhaps the most troubling from the perspective of random probability analysis and is expected to have higher random rates because of only one position correlation. However, the shortness of time between events tends to make the randomness lower (see Section 4 for results). The half-life of ${ }^{284} \mathrm{Fl}$ is estimated to be $2.8_{-1.1}^{+5.1} \mathrm{~ms}$.

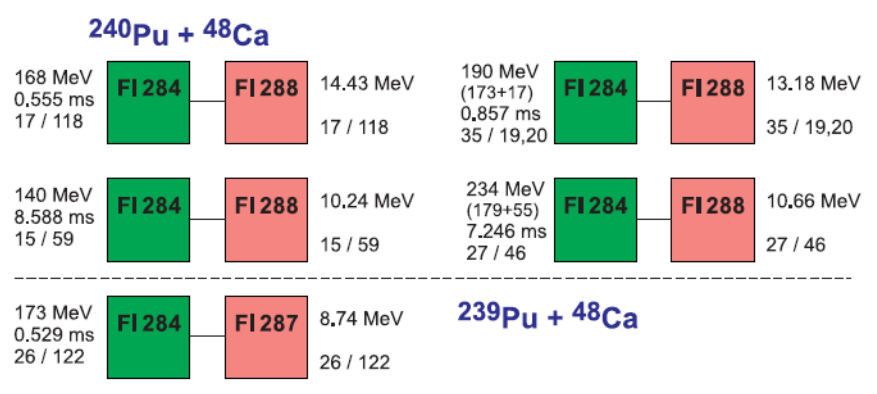

Figure 3: Decay properties of nuclei assigned to ${ }^{284} \mathrm{Fl}$ observed in the ${ }^{48} \mathrm{Ca}+{ }^{240} \mathrm{Pu}$ (top) and ${ }^{48} \mathrm{Ca}+{ }^{239} \mathrm{Pu}$ (bottom) reactions. The right rows for each chain show ER (in pink) energies and strip numbers (front and back). The left rows provide SF fragment (in green) energies, time intervals between each event, and their strip numbers. The decay chain shown in the top right corner was registered in two back strips. This figure is reproduced from Figure 1 in [7].

\section{3. ${ }^{48} \mathbf{C a}+{ }^{251} \mathbf{C f}$ experiments}

Experiments aimed at the synthesis of heavier isotopes of Og were performed using ${ }^{48} \mathrm{Ca}$ beams and ${ }^{251} \mathrm{Cf}$ (mixed) target and are discussed in more detail in Brewer, et al. [9]. The experiments were performed at the DGFRS using the ${ }^{48} \mathrm{Ca}$ beam accelerated at the U400 cyclotron of the Flerov Laboratory of Nuclear Reactions, JINR as described in Section 2.

The target materials were provided by Oak Ridge National Laboratory (ORNL) salvaged from old ${ }^{252} \mathrm{Cf}$ sources and contained multiple $\mathrm{Cf}$ isotopes with the following weight percentages at the time of target manufacture: $50.7 \%{ }^{249} \mathrm{Cf}, 12.9 \%{ }^{250} \mathrm{Cf}, 36.4 \%{ }^{251} \mathrm{Cf}$, and $0.02 \%{ }^{252} \mathrm{Cf}$. Target sectors were manufactured at ORNL and shipped to Dubna to assemble a standard target wheel. The average thicknesses of the targets were $0.35 \pm 0.06 \mathrm{mg} / \mathrm{cm}^{2}$. The targets were manufactured at ORNL by depositing $\mathrm{CfO}_{2}$ oxide onto $0.71-0.72 \mathrm{mg} / \mathrm{cm}^{2}$ Ti foils. The irradiation with ${ }^{48} \mathrm{Ca}$ was similarly performed at two beam energies of $252 \mathrm{MeV}$ and $258 \mathrm{MeV}$ as described in Section 2 and occurred between Oct. 2015 and April 2016. A total beam dose of $1.6 \times 10^{19}$ and $1.1 \times 10^{19}$ 
particles, respectively, was obtained. The experiment was discontinued because beam-induced target contamination altered the thickness of the target thus reducing recoils of products into the DGFRS.

Four decay chains were observed for ${ }^{294} \mathrm{Og}$ from past ${ }^{48} \mathrm{Ca}$ irradiations of ${ }^{249} \mathrm{Cf}$ [10] and ${ }^{249} \mathrm{Bk}$ [11] targets (from the in-growth of ${ }^{249} \mathrm{Cf}$ ). The analog data collected during the ${ }^{48} \mathrm{Ca}+$ ${ }^{251} \mathrm{Cf}$ experiment was analyzed to determine the random probability of ${ }^{294} \mathrm{Og}$-like decay chains observed in the prior experiments and as shown in Fig. 1. The results of this analysis are presented in Section 4.

\section{Monte Carlo Random Probability (MCRP) analysis}

The Monte Carlo Random Probability (MCRP) method is described in more detail in references $[5,6]$. This method uses the actual data collected during an experiment and the same search technique used to identify correlated events making an interesting decay chain or sequence. Random events are inserted into the data, and the search algorithm is run again to find any correlation that might be in the data, but now correlated with a random event. The events must be within the same front and back strips (vertical and horizontal position) and within a given time window in order to be correlated. Because the actual data is used, this method automatically includes fluctuations in background, counting rates, detector performance, beam rates, cosmic ray rates, high voltage drifts, gain shifts, etc., that may occur during an experiment running 24 hours a day for months at a time. Thus, stretches of data that are noisy might be expected to exhibit higher random correlation rates and those that are quiescent might be expected to have lower random correlation rates compared to a method using average rates of various events occuring during the course of the experiment. The MCRP method was tested with a variety of random number generators, distributions of random events in the detector system, and a variety of other factors which are detailed in references [5, 6] and found to be relatively insensitive to these factors. For this study, a flat distribution of random events was inserted into the detector with no attempt made to mimic the distribution of ERs implanted into the detector. The various experiments were studied independently. Typically $1 \times 10^{9}$ or more random events were inserted into the data. Random probabilities below $1 \times 10^{-9}$ were obtained by extrapolation or multiplication of various pairs of correlated random probabilities.

A comparison of the MCRP method with the Schmidt method [12] for the ${ }^{48} \mathrm{Ca}+{ }^{239} \mathrm{Pu}$ experiment is shown in Table 1. Similar energy and position correlation windows were used for both methods and the correlation time was $1 \mathrm{~s}$. Several items should be noted from Table 1. The Schmidt method based on average rates yields identical random probability values for similar decay chains regardless of where the random event is inserted - for example, decay chains of the type A-A-R, A-R-A and R-A-A all have the same probability of $2.4 \times 10^{-9}$, whereas the MCRP method takes into account the distribution of events in time also and thus yields random probabilities with different values of $2.0 \times 10^{-9}, 5.4 \times 10^{-8}$ and $7.3 \times 10^{-9}$, respectively. Note that the MCRP value is higher in all cases where there are three or more events, leading to the conclusion that the probability calculated with this method is slightly more conservative. The longer decay chains have much lower probabilities that are due to random events in both methods. The ER-F decay observed in the ${ }^{48} \mathrm{Ca}+{ }^{239,240} \mathrm{Pu}$ experiments that has been assigned to ${ }^{284} \mathrm{Fl}$ is the decay chain of most concern. 


\begin{tabular}{ccc} 
Correlation & MCRP method & Schmidt method [12] \\
\hline ER-R & $2.6 \times 10^{-6}$ & $4.9 \times 10^{-5}$ \\
A-R & $3.6 \times 10^{-6}$ & $4.9 \times 10^{-5}$ \\
R-A & $3.4 \times 10^{-6}$ & $4.8 \times 10^{-5}$ \\
R-F & $9.6 \times 10^{-8}$ & $5.7 \times 10^{-7}$ \\
ER-A-R & $5.8 \times 10^{-8}$ & $2.4 \times 10^{-9}$ \\
ER-R-A & $6.6 \times 10^{-8}$ & $2.4 \times 10^{-9}$ \\
ER-R-F & $3.7 \times 10^{-9}$ & $2.8 \times 10^{-11}$ \\
A-A-R & $2.0 \times 10^{-9}$ & $2.4 \times 10^{-9}$ \\
A-R-A & $5.4 \times 10^{-8}$ & $2.4 \times 10^{-9}$ \\
A-R-F & $1.4 \times 10^{-10}$ & $2.8 \times 10^{-11}$ \\
R-A-A & $7.3 \times 10^{-9}$ & $2.4 \times 10^{-9}$ \\
R-A-F & $9.1 \times 10^{-10}$ & $2.8 \times 10^{-11}$
\end{tabular}

Table 1: Comparison of the MCRP method described in this paper with the Schmidt method [12] based on average rates of various types of events occuring in the detector. Similar energy windows and position correlation windows were used for both methods and the correlation time was $1 \mathrm{~s}$. Evaporation residues (ER), alpha-particles (A), fission fragments (F) and random (R) events are correlated in various combinations.

The relatively short life-time of ${ }^{284} \mathrm{Fl}$ of $2.8 \mathrm{~ms}$ should also result in a very low random probability. The results in Table 1 are for correlation times of $1 \mathrm{~s}$; when this time is reduced to $1 \mathrm{~ms}$, the MCRP result is a lower probability of $1.8 \times 10^{-10}$. We conclude that the ER-F events observed in the ${ }^{48} \mathrm{Ca}$ $+{ }^{239,240} \mathrm{Pu}$ experiments are highly unlikely to be random.

The long decay chains originating from ${ }^{285} \mathrm{Fl}$ shown in Figure 2 from the ${ }^{48} \mathrm{Ca}+{ }^{240} \mathrm{Pu}$ experiments were also analyzed for the probability that they were random using the MCRP method. The number of these correlated decay chains being due to random events was estimated to be less than $5 \times 10^{-17}$. Because this probability is extremely low, the randomness of these events are not discussed further.

The MCRP results for about half of the data (the $252-\mathrm{MeV}$ beam energy) for the ${ }^{48} \mathrm{Ca}+{ }^{251} \mathrm{Cf}$ experiment are shown in Table 2 as a function of correlation time. Note the increase in probability as the correlation time increases from $0.1 \mathrm{~s}$ to $5 \mathrm{~s}$. The MCRP probability that a ${ }^{294} \mathrm{Og}$-like decay chain occurs in the ${ }^{48} \mathrm{Ca}+{ }^{251} \mathrm{Cf}$ experiment is $1.8 \times 10^{-14}$ and indicates that such a decay chain would be highly unlikely to be due to random events.

\section{Conclusions and Future Work}

Experimental evidence was presented for the observation of a new isotope of $\mathrm{Fl},{ }^{284} \mathrm{Fl}$, as well as additional decay chains were observed for ${ }^{285} \mathrm{Fl}$ from ${ }^{48} \mathrm{Ca}$ induced nuclear reactions on lighter ${ }^{239,240} \mathrm{Pu}$ targets. A total of four atoms of ${ }^{294} \mathrm{Og}$ have been observed in several experimental runs (three using a ${ }^{249} \mathrm{Cf}$ target, and one using a ${ }^{249} \mathrm{Bk}$ target that had significant in-growth of ${ }^{249} \mathrm{Cf}$. Monte Carlo Random Probability analysis indicates that these observed decay chains are highly unlikely to be due to random events. The MCRP method and Schmidt [12] methods are in agreement, with the MCRP method being more conservative for decay chains longer than one decay. The low random probability for a ${ }^{294} \mathrm{Og}$-like decay chain indicates that it is highly unlikely 


\begin{tabular}{cccc} 
Correlation & $0.1 \mathrm{~s}$ & $1 \mathrm{~s}$ & $5 \mathrm{~s}$ \\
\hline R-F & $6.3 \times 10^{-9}$ & $8.3 \times 10^{-8}$ & $3.8 \times 10^{-7}$ \\
R-A & $2.5 \times 10^{-6}$ & $2.4 \times 10^{-5}$ & $1.0 \times 10^{-4}$ \\
A-R & $3.2 \times 10^{-6}$ & $2.7 \times 10^{-5}$ & $1.4 \times 10^{-4}$ \\
R-A-A-F* & $1.8 \times 10^{-14}$ & $2.2 \times 10^{-12}$ & $4.6 \times 10^{-11}$
\end{tabular}

${ }^{*}$ These values were calculated from random probabilities determined for decay chain fragments and multiplication of the appropriate probabilities together for this longer decay chain.

Table 2: MCRP method calculated for varying correlation times for a ${ }^{294} \mathrm{Og}$-like decay chain shown in Figure 1 using about half of the ${ }^{48} \mathrm{Ca}+{ }^{251} \mathrm{Cf}$ experimental data. Evaporation residues (ER), alpha-particles $(\mathrm{A})$, fission fragments $(\mathrm{F})$ and random $(\mathrm{R})$ events are correlated in various combinations.

to be due to random events. Future work at DGFRS will include the attempt to produce lighter isotopes of $\mathrm{Fl}$ using the ${ }^{48} \mathrm{Ca}+{ }^{240} \mathrm{Pu}$ reaction at higher beam energies and the attempt to observe an alpha-decay branch of ${ }^{284} \mathrm{Fl}$.

\section{Acknowledgements}

The authors would like to acknowledge that some of this work was performed by the DubnaLLNL-ORNL-VU collaboration and the valuable discussions held within the collaboration. The ${ }^{240} \mathrm{Pu}$ used in this research was partially supplied by the United States Department of Energy Office of Science by the Isotope Program in the Office of Nuclear Physics.

These studies were supported by the Russian Foundation for Basic Research, including recent Grants No. 13-02-12052 and No. 13-03-12205, and by the Moscow Region Government through Grant No. 736/36-16.09.2014. Research at ORNL was supported by the U.S. DOE Office of Nuclear Physics under DOE Contract No. DE-AC05-00OR22725 with UTBattelle, LLC. Research at LLNL was supported by LDRD Program Project No. 08-ERD-030, under DOE Contract No. DE-AC52-07NA27344 with Lawrence Livermore National Security, LLC. This work was also supported by the U.S. DOE through Grant No. DE-FG-05-88ER40407 (Vanderbilt University). SYS was also supported by the Stewardship Science Graduate Fellowship through Grant DE-NA0002135.

\section{References}

[1] Yu. Ts. Oganessian, et al., J. Phys. G 34, R165 (2007), and references therein.

[2] Yu. Ts. Oganessian, et al., Phys. Rev. Lett. 109, 162501 (2012).

[3] Yu. Ts. Oganessian, et al., Prog. Theor. Phys. Supp. 154, 406 (2004).

[4] Yu. Ts. Oganessian, et al., Phys. Rev. C 62, 041604 (2000).

[5] N.J. Stoyer, et al., Nucl. Instrum. Methods Phys. Res. A 455, 433 (2000).

[6] M.A. Stoyer, et al., Eur. J. Phys. A 25, 595 (2005).

[7] V.K. Utyonkov, et al., Phys. Rev. C 92, 034609 (2015).

[8] P.A. Ellison, et al., Phys. Rev. Lett. 105, 182701 (2010). 
[9] N. Brewer, et al., in preparation for Phys. Rev. C (2017).

[10] Yu. Ts. Oganessian, et al., Phys. Rev. C 74, 044602 (2006).

[11] Yu. Ts. Oganessian, et al., Phys. Rev. C 83, 054315 (2011).

[12] K.-H. Schmidt, et al., Z. Phys. A 316, 19 (1984). 